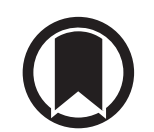

CrossMark

\title{
Innate lymphoid cells in the upper airways: importance of CD117 and IL-1RI expression
}

\author{
Koen Van Crombruggen ${ }^{1}$, Sylvie Taveirne ${ }^{2}$, Gabriele Holtappels ${ }^{1}$, \\ Georges Leclercq ${ }^{2}$ and Claus Bachert ${ }^{1,3}$
}

Affiliations: 'Upper Airway Research Laboratory, Dept of Otorhinolaryngology, Ghent University Hospital, Ghent, Belgium. 'Laboratory of Experimental Immunology, Ghent University, Ghent, Belgium. ${ }^{3}$ Division of ENT Diseases, CLINTEC, Karolinska Institute, University of Stockholm, Stockholm, Sweden.

Correspondence: Koen Van Crombruggen, Upper Airway Research Laboratory, Dept of Otorhinolaryngology, Ghent University Hospital, De Pintelaan 185, 9000 Ghent, Belgium. E-mail: koen.vancrombruggenđugent.be

@ERSpublications

CD117 expression in human ILC2s depends on the local tissue milieu, and IL-1RI expression is not ubiquitous in ILC2s http://ow.ly/Iea230mcKz5

Cite this article as: Van Crombruggen K, Taveirne S, Holtappels G, et al. Innate lymphoid cells in the upper airways: importance of CD117 and IL-1RI expression. Eur Respir J 2018; 52: 1800742 [https://doi. org/10.1183/13993003.00742-2018].

ABSTRACT Although type 1, 2 and 3 innate lymphoid cells (ILC1s, ILC2s and ILC3s, respectively) are emerging as important cell populations regulating tissue homeostasis, remodelling and inflammation, a vast majority of our knowledge stems from in vitro and murine experiments, and requires thorough confirmation in human diseases.

Relative levels of ILCs were evaluated by means of flow cytometry in freshly resected human upper airways mucosa of patients with chronic rhinosinusitis without nasal polyps (CRSsNP) and with nasal polyps (CRSwNP), taking into account the patient's clinical parameters and disease comorbidities.

We report that the CD117 and interleukin-receptor type I (IL-1RI) expression status of human ILC2s depends on the local tissue environment. Only CD117 ${ }^{+} \mathrm{IL}_{-1 R I^{+}} \mathrm{ILC} 2 \mathrm{~s}$, exclusively present in CRSwNP, possess an interrelationship with type 2 T-helper cell cytokine and eosinophil levels in human upper airway mucosa. In CRSsNP, mainly CD117 ${ }^{-} \mathrm{IL}-1 \mathrm{RI}^{-}$ILC2s are increased, yielding lower eosinophilia in this disease despite the high levels of ILC2s.

These data unveil that the $\mathrm{CD} 117^{-}$and $\mathrm{CD} 117^{+}$fractions within the native human ILC2 population are not a random phenomenon, in contrast to what could be concluded from in vitro data, and that the IL-1RI expression is not ubiquitous in ILC2s in vivo in humans, which cannot be assessed via in vitro and murine experiments.

This article has supplementary material available from erj.ersjournals.com

Received: April 192018 | Accepted after revision: Oct 032018

Copyright OERS 2018 


\section{Introduction}

Chronic rhinosinusitis (CRS) is a heterogeneous group of chronic airway inflammatory diseases affecting the mucosa of the nasal and paranasal cavities and is either accompanied by nasal polyp formation (CRSwNP) or presents without polyps (CRSsNP) [1]. CRS shows a high prevalence of up to $15 \%$ in adults, often leading to the subsequent development of lower respiratory tract conditions, especially asthma [2]. CRSsNP and CRSwNP are distinct disease entities, with CRSwNP being associated with a T-helper type 2 (Th2)-skewed eosinophilic inflammation with high interleukin (IL)-5 and eosinophil cationic protein (ECP) concentrations in the polyps, while CRSsNP is characterised by a more predominant Th1 milieu with pronounced levels of interferon (IFN)- $\gamma$ in the inflamed ethmoidal mucosa [1]. Furthermore, novel evidence indicates considerable heterogeneity within the CRSwNP subgroup, determining the risk of comorbid asthma. Despite continuous progress in research, which contributes to the further unveiling of the pathophysiology of these chronic airway conditions, the aetiology remains poorly understood and appears to be multifactorial.

Over the past years a new class of innate effector cells whose development relies on signalling through the IL-2 receptor common $\gamma$ chain and IL-7R $\alpha$ (CD127) has drawn much attention. These cells are referred to as helper innate lymphoid cells (ILCs) [3-5]. In contrast to T- and B-cells, this ILC lineage does not express recombined, antigen-specific receptors and is substantially represented at barrier surfaces. Based on the expression of lineage-defining transcription factors and on their functional similarities to Th-cells, three subsets of helper ILCs can be discriminated, being arranged over three global ILC groups.

Group 1 ILCs include besides an IFN- $\gamma$-secreting helper ILC subset, referred to as ILC1s, the cytotoxic conventional natural killer cells and intraepithelial type 1 innate lymphoid cells. ILC1s produce IFN- $\gamma$ on activation with IL-12, IL-15, and IL-18. They have been shown to be implicated in the defence against viruses, bacteria and protozoa. ILC1s are reported to be predominantly present in chronically inflamed mucosal tissues, being absent under normal physiology [6]. Group 2 ILCs currently only consists of ILC2s that secrete the typical Th2 cell-associated cytokines IL-4, IL-5, IL-9 and IL-13. They produce amphiregulin, a member of the epidermal growth factor family involved in limiting and repairing tissue damage. ILC2s are involved during early immune responses to helminth infection, but are also important for allergen-induced airway inflammation and tissue repair. ILC2s can be activated by various cytokines, such as IL-33, IL-25, thymic stromal lymphopoietin (TSLP) and IL-1 $\beta$, as well as lipid mediators such as eicosanoids, including prostaglandin D2 and leukotriene D4 [7]. Group 3 ILCs comprise different populations including lymphoid tissue inducer (LTi) cells, natural cytotoxicity receptor (NCR) ${ }^{+}$ILC3s and $\mathrm{NCR}^{-}$ILC3s. The $\mathrm{NCR}^{+}$and $\mathrm{NCR}^{-}$ILC3 fractions produce IL-22 and IL-17, respectively, and are regarded as defenders of mucosal barrier function implicated in immunity against extracellular bacteria and autoimmune diseases. Both cell populations produce cytokines upon activation by IL-1 $\beta$ and IL-23 [4, 5]. LTi cells are important in formation of lymph nodes during fetal development [8].

Upon their initial characterisation, ILC2s were reported to be abundantly present in the upper airway mucosa of CRSwNP patients [9], and sinonasal tissues became popular to translate mechanistic findings into the research of ILCs. However, most of the translational parts of these studies were performed on a low number of patients reporting no or limited clinical data, and consequently not taking into account the potential effect of comorbidities [9-11]. Moreover, the presence of the other ILC subpopulations in upper airway mucosa of CRSsNP patients has received less attention.

In the present report we show that in noninflamed conditions, ILC1s are the most prominent ILCs in sinonasal tissue. In CRSwNP, ILC1s are decreased, while ILC2s per se are not increased. Focusing on CD117 $7^{+}$and IL-1RI ${ }^{+}$fractions within the ILC2s, and taking comorbidities into account, we unveil the precise contribution of ILC2s in CRSwNP; we describe an explicit increase in CD117 ${ }^{+} \mathrm{IL}_{-1 R I^{+}}$ILC2s levels in CRSwNP, showing an interrelationship with Th2 cytokine and eosinophil levels. In CRSsNP, mainly CD117 ${ }^{-} \mathrm{IL}^{-1 \mathrm{RI}^{-}} \mathrm{ILC}_{2 \mathrm{~s}}$ are increased, yielding lower eosinophilia in this disease, despite the high levels of total ILC2s.

\section{Methods}

Patients

The patients' clinical data are described in table 1. More detailed information can be found in the supplementary material.

\section{Flow cytometry staining}

Tissue native ILCs were gated as living, lymphogate $\mathrm{CD}^{+} 5^{+}$lineage negative $\left(\mathrm{CD} 3{ }^{-} \mathrm{CD} 11 \mathrm{c}^{-}\right.$ $\left.\mathrm{CD} 14^{-} \mathrm{CD} 19^{-} \mathrm{CD} 34^{-} \mathrm{BDCA}^{-}{ }^{-} \mathrm{FcER} 1^{-} \mathrm{CD} 94^{-} \mathrm{CD} 1 \mathrm{a}^{-} \mathrm{CD} 123^{-} \mathrm{TCR} \alpha \beta^{-}\right)$cells. ILC1 were further phenotyped

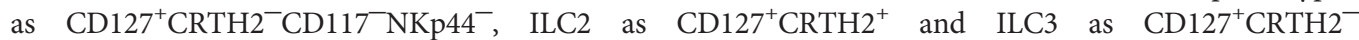
$\mathrm{CD} 117^{+} \mathrm{IL}-1 \mathrm{RI}^{+} \mathrm{NKp} 44^{-}$for the $\mathrm{NCR}^{-}$fraction and $\mathrm{CD} 127^{+} \mathrm{CRTH} 2^{-} \mathrm{CD} 117^{+} \mathrm{IL}-1 \mathrm{RI}^{+} \mathrm{NKp} 44^{+}$for the $\mathrm{NCR}^{+}$fraction, based on a previously reported gating strategy [12] and according to the gating strategy 


\begin{tabular}{|c|c|c|c|}
\hline & Control & CRSsNP & CRSwNP \\
\hline Subjects & 29 & 17 & 35 \\
\hline Age years & $32(18-59)$ & 41 (19-63) & $44(24-68)$ \\
\hline Male/female & $13 / 11$ & $7 / 10$ & $21 / 14$ \\
\hline Smokers & 7 & 2 & 0 \\
\hline Allergy $^{+}$asthma $^{-}$ & 6 & 5 & 8 \\
\hline Allergy $^{-}$asthma ${ }^{+}$ & 6 & 0 & 6 \\
\hline Allergy $^{+}$asthma ${ }^{+}$ & 5 & 5 & 9 \\
\hline AERD & 0 & 0 & 0 \\
\hline
\end{tabular}

Data are presented as $\mathrm{n}$, or median (range). CRSsNP: chronic rhinosinusitis without nasal polyps; CRSwNP: chronic rhinosinusitis with nasal polyps; AERD: aspirin-exacerbated respiratory disease.

shown in supplementary figure E1. The levels of the different ILC subpopulations are expressed as percentage of the total living cells. Further methodological details are described in the supplementary material.

In vitro assessment of the functional responses by freshly isolated ILC2s

The single cells were resuspended in tissue culture medium supplemented with IL-2 $\left(5 \mathrm{ng} \cdot \mathrm{mL}^{-1}\right)$. The cells were activated with $50 \mathrm{ng} \cdot \mathrm{mL}^{-1} \mathrm{PMA}$ (Sigma, St Louis, MO, USA) and $1 \mu \mathrm{g} \cdot \mathrm{mL}^{-1}$ ionomycin (Sigma) for $3 \mathrm{~h}$ at $37^{\circ} \mathrm{C}$ in the presence of $5 \%$ carbon dioxide. $1 \mathrm{~h}$ after the start of the PMA/ionomycin treatment, brefeldin A $\left(5 \mu \mathrm{g} \cdot \mathrm{mL}^{-1}\right)$ (eBioscience, Waltham, MA, USA) was added. The samples were subjected to flow cytometry staining as described earlier and in the supplementary material.

\section{Tissue homogenates}

Frozen tissue samples from the same subject used for ILC flow cytometry were homogenised by means of mechanical disruption as described in the supplementary material.

\section{Measurement of inflammatory mediators}

The inflammatory mediators IL-4, IL-5, IL-12 and IL-25 were measured on tissue homogenates by means of Luminex xMAP technology using the Fluorokine MAP Multiplex Human Cytokine kit (R\&D Systems, Minneapolis, MN, USA) on a Bio-PlexTM 200 Array Reader (Bio-Rad, Hercules, CA, USA). Tissue ECP levels were measured using UniCAP (Phadia, Uppsala, Sweden)

\section{Statistical analysis}

Statistical analysis was performed by nonparametric Mann-Whitney U-test. A p-value $<0.05$ was considered to be statistically significant (GraphPad, San Diego, CA, USA).

\section{Results}

Relative levels of ILCS in human upper airway mucosa of controls, CRSsNP and CRSWNP

Because the levels of $\mathrm{CD} 45^{+}$cells were higher in CRSsNP and CRSwNP than in controls (supplementary figure E2A), while the number of total livings cell were similar between the different subject groups (supplementary figure E2B), the flow cytometry data were expressed as a percentage of total living cells. In order to allow the reader to compare the levels of the different ILC groups to other literature sources where ILCs might be expressed as a percentage of $\mathrm{CD} 45^{+}$cells, in this section the results are presented in both ways [9-11].

Members of all three ILC groups were present in human upper airway mucosa of all patient groups. In noninflamed control conditions, ILC1s represent the most prominent subset, followed by $\mathrm{NCR}^{-}$ILC3s, while ILC2s and $\mathrm{NCR}^{+}$ILC3s showed the weakest abundance (figure 1 and supplementary figure E3). In the CRSsNP patient group, a significant increase in ILC2 and NCR ${ }^{-}$ILC3 populations was observed compared to the mucosa of control subjects, whereas the levels of ILC1 and NCR ${ }^{+}$ILC3 cells were similar (figure 1 and supplementary figure E3). The inflamed mucosa of patients with CRSwNP contained significant lower levels of ILC1s compared to noninflamed control mucosa, while the ILC2 and NCR ILC3 populations were significantly increased. $\mathrm{NCR}^{+}$ILC3 cells maintained a low level of abundance, which was similar to control mucosa (figure 1 and supplementary figure E3). 


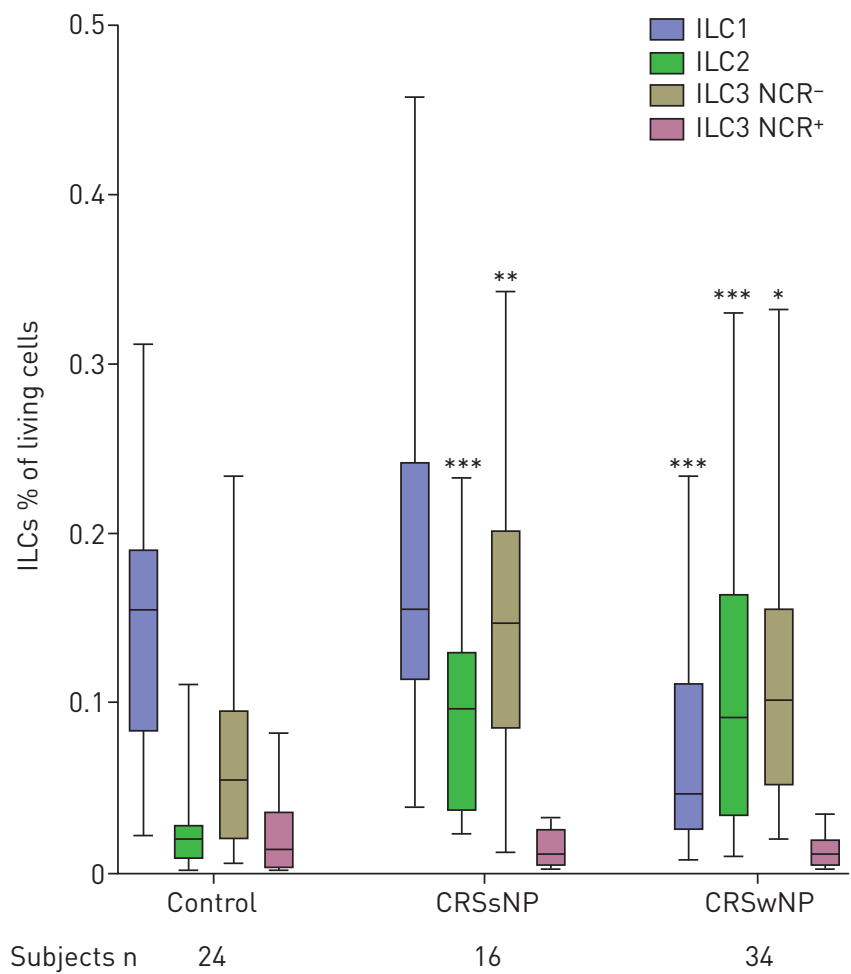

FIGURE 1 Relative number of the three innate lymphoid cell (ILC) fractions in controls, patients with chronic rhinosinusitis without nasal polyps (CRSsNP) and with nasal polyps (CRSwNP). Values are expressed as percentage ILCs of total living cells, and presented as box-and-whisker plots showing the minimum and the maximum value, the lower and the upper quartile, and the median. NCR: natural cytotoxicity receptor. ${ }^{*}: p<0.05 ;{ }^{* *}: p<0.01 ;{ }^{* * *}$ : $p<0.001$ versus controls.

ILCS in upper airway mucosa of controls, CRSSNP and CRSWNP: the effect of comorbidities As the frequently occurring CRS comorbidities allergy and asthma can potentially affect the levels of the ILC subsets in the mucosa, we also took these comorbidities into account.

Within the noninflamed upper airway mucosa of control individuals and within the CRSsNP patient group, the presence of allergy, asthma and allergic asthma had no influence on the number of any ILC subset (figures 2-4).

The significant reduction in the levels of ILC1 cells that was observed in the undifferentiated CRSwNP patient group (figure 1) can be attributed to the reduced numbers of ILC1s in CRSwNP patients without comorbidities and those suffering from comorbid allergy or comorbid asthma (figure 2). However, and importantly, CRSwNP patients with comorbid allergic asthma showed no reduction in levels of ILC1 compared to the equivalent control patients with allergic asthma but without chronically inflamed upper airway mucosa (figure 2).

Unexpectedly, the levels of ILC2s were also significantly higher in the Th1 cytokine-skewed disease CRSsNP in comparison to the respective control patient groups. Moreover, the different comorbidities had no effect on the levels of ILC2 cells (figure 3). ILC2s are not involved in the pathophysiology of CRSwNP, because the inflamed mucosa of CRSwNP patients without comorbidities contained a similar amount of ILC2s compared to noninflamed mucosa of control subjects (figure 3). The levels of ILC2s were clearly increased in patients with comorbid allergy and asthma, with allergic asthma being the most pronounced (figure 3).

While the NCR ${ }^{-}$ILC3 levels were slightly but significantly increased in the undifferentiated CRSsNP and CRSwNP patient groups compared to controls (figure 1), when comorbidities were taken into account, a variable outcome could be observed only yielding statistical significant higher levels for CRSsNP patients without comorbidities and CRSwNP with comorbid asthma (figure 4a). The levels of NCR ${ }^{+}$ILC3 overall remained very low in all patient groups with no influence by comorbidities (figure 4b).

\section{CD117 and IL-1RI expression in the ILC2 population}

Next, we assessed the relative levels of CD117-positive and -negative fractions within the ILC2 population $[3,5,9]$ and investigated the expression of IL-1RI on ILC2 cells in view of the contrasting reports present in the literature $[5,10,13-15]$. 


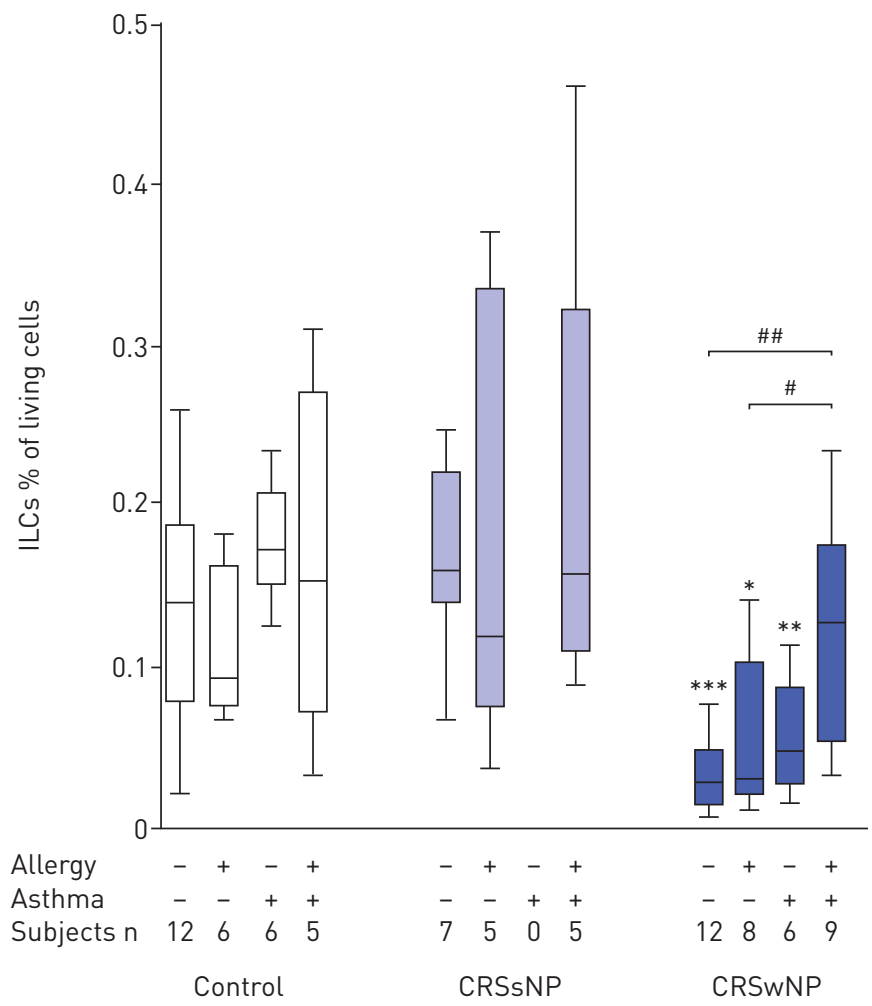

FIGURE 2 Relative number of type 1 innate lymphoid cell (ILC1s) in controls, patients with chronic rhinosinusitis without nasal polyps (CRSsNP) and with nasal polyps (CRSwNP) separated on comorbidity status. Data are presented as percentage ILC1s of total living cells, and presented as box-and-whisker plots showing the minimum and the maximum value, the lower and the upper quartile and the median. $-:$ absent; +: present. ${ }^{*}: \mathrm{p}<0.05 ;{ }^{* *}: \mathrm{p}<0.01 ;{ }^{* * *}: \mathrm{p}<0.001$ versus controls; ${ }^{\#}: \mathrm{p}<0.05 ;{ }^{\# \#}: \mathrm{p}<0.01$ versus the indicated subject group.

Regardless of the presence or absence of allergy, asthma or allergic asthma, within the control subjects and the CRSsNP patient group, the majority of the ILC2 cells were CD117 ${ }^{-}$, being statistically significant higher than the CD117 cells; the latter virtually being absent (figure 5a). In contrast, in the inflamed mucosa of CRSwNP without comorbidities, the CD117 ${ }^{+}$ILC2 fraction was increased, while the CD117 fraction remains on the level found in controls, yielding two fractions lacking statistical differences (figure 5a). The levels of both $\mathrm{CD} 117^{+}$and CD117 $7^{-}$ILC2 fractions in CRSwNP further increased under the influence of comorbid allergy and asthma (figure $5 \mathrm{~b}$ and $\mathrm{c}$ ), maintaining a similar number of cells in both fractions, except for the mucosa of CRSwNP patients with comorbid allergic asthma, which possesses significantly higher numbers of CD117 ${ }^{-}$ILC2 compared to CD $117^{+}$cells (figure 5a).

Similar to that observed for CD117, within the control subjects and the CRSsNP patient group, the majority of the ILC2 cells belonged to the IL-1RI ${ }^{-}$fraction. This fraction was consistently significantly more pronounced than the IL-1RI ${ }^{+}$fraction, which was virtually absent (figure 6a). The IL-1RI ${ }^{-}$ILC2 fraction was not affected by comorbid allergy and/or asthma (figure 6b). For CRSwNP, the number of IL-1RI ${ }^{+}$cells was clearly higher in patients with comorbid allergy and/or asthma (figure 6c), ending up with similar levels of IL-1RI ${ }^{+}$and IL1R ${ }^{-}$ILC2s in CRSwNP patients with allergy or with asthma (figure 6a). Remarkably, the effect of allergy and asthma on the level of IL-1RI ${ }^{+}$ILC2s was not additive in allergic asthmatics, showing similar levels as observed for allergic CRSwNP and asthmatic CRSwNP patients (figure 6c), while the levels of IL-1RI- ILC2s were increased in patients with comorbidities, being most pronounced in allergic asthma (figure $6 \mathrm{~b}$ ). Neither IL-1RI ${ }^{-}$nor IL-1RI ${ }^{+}$ILC2 cells were manifestly increased as a consequence of the pathological process of CRSwNP without comorbidities (figure $6 \mathrm{~b}$ and c).

Representative figures of the gating step assessing the expression of CD117 and IL1RI on ILC2 cell population in controls, CRSsNP and CRSwNP all suffering from comorbid allergic asthma are shown in supplementary figure E4. Both the $\mathrm{CD} 117^{+}$and $\mathrm{CD} 117^{-}$ILC2 fraction contained IL-1RI ${ }^{+}$and IL-1RI subpopulations (supplementary figure E5).

In vitro assessment of the functional responses by freshly isolated ILC2S

Details are provided in the supplementary material. 


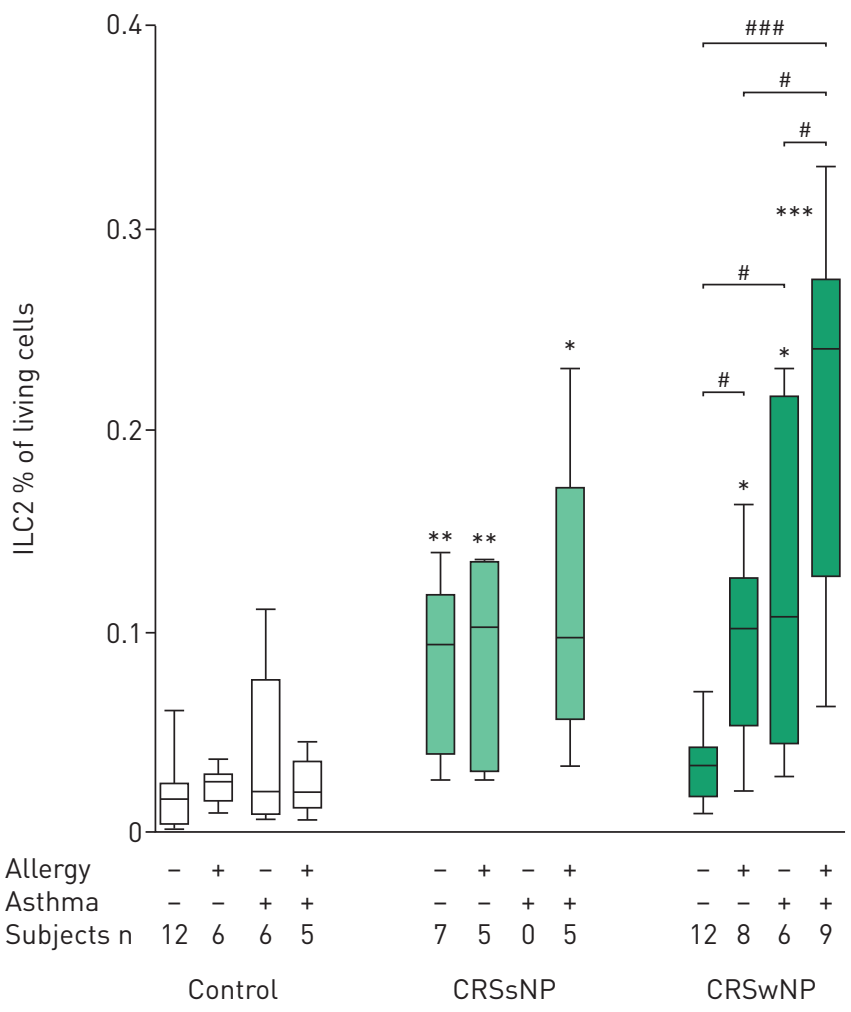

FIGURE 3 Relative numbers of type 2 innate lymphoid cells (ILC2s) in controls, patients with chronic rhinosinusitis without nasal polyps (CRSsNP) and with nasal polyps (CRSwNP) separated on comorbidity status. Data are presented as percentage ILC2s of total living cells, and presented as box-and-whisker plots showing the minimum and the maximum value, the lower and the upper quartile, and the median. $-:$ absent; + : present. ${ }^{*}: p<0.05 ;{ }^{* *}: p<0.01 ;{ }^{* * *}: p<0.001$ versus controls; ${ }^{\#}: p<0.05 ;{ }^{\# \# \#}: p<0.001$ versus the indicated subject group.

\section{Tissue cytokine levels}

Levels of IL-4 were slightly higher in CRSsNP and CRSwNP compared to controls, not reaching statistical significance (figure 7a). Within the CRSwNP patient group, the levels of IL-4 were similar for each comorbidity status (figure 7a), consequently showing no parallelism with the number of ILC2s in CRSwNP (figures 3, 5 and 6).

IL-5 levels were significantly increased in all CRSwNP comorbidity subgroups versus the corresponding control subject groups. Within the CRSwNP patients, subjects with comorbid allergy, asthma and allergic asthma had significantly higher IL-5 levels than patients without these comorbidities. CRSsNP patients showed no significant differences (figure $7 \mathrm{~b}$ ).

Tissue ECP levels were significantly higher in all CRSwNP comorbidity subgroups in comparison to the corresponding control subject groups. Within the CRSwNP patients, subjects with comorbid allergy, asthma and allergic asthma had significantly higher ECP levels than patients without these comorbidities. The levels observed in CRSsNP were also increased versus controls but only reaching significance for the patient group without comorbidities (figure 7c).

IL-25 was only measurable in CRSwNP, yielding similar levels for every comorbidity status (figure 7d). IL-12 was below the detection limit for all samples (data not shown).

\section{Discussion}

The observation that from the three ILC groups present, ILC1s are the most abundant in noninflamed tissues argues against the literature dogma that ILC1 cells are exclusively present under inflammatory conditions [6]. While this might be true for gastrointestinal tissues [16, 17], this appears not to be transposable to the airway mucosa. Moreover, the opposite seems to be true because the inflamed CRSwNP mucosa shows lower levels of ILC1s than control mucosa. As CRSwNP is particularly known as a Th2 cytokine-skewed eosinophilic inflammatory condition with high IL-5 and ECP concentrations in the polyps [1], reduced ILC1 levels seem to be consistent with this. However, while in the undifferentiated CRSwNP group this Th2 cytokine signature could be further corroborated by accompanying increased 

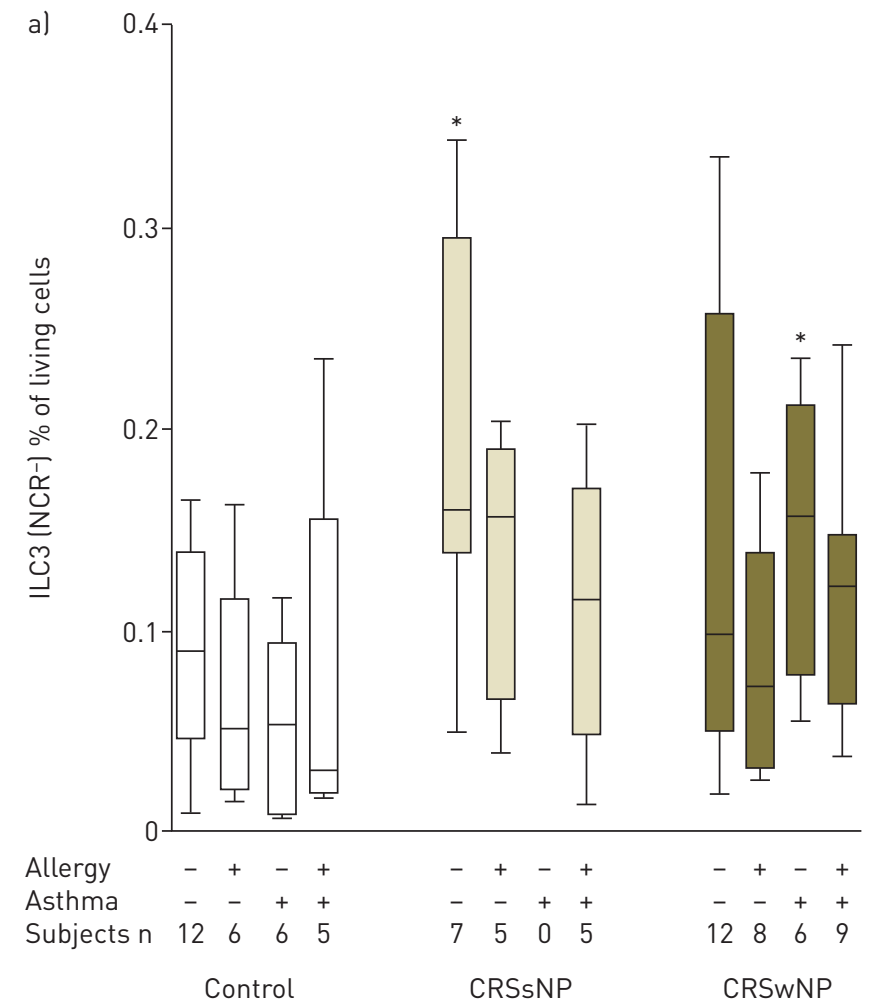

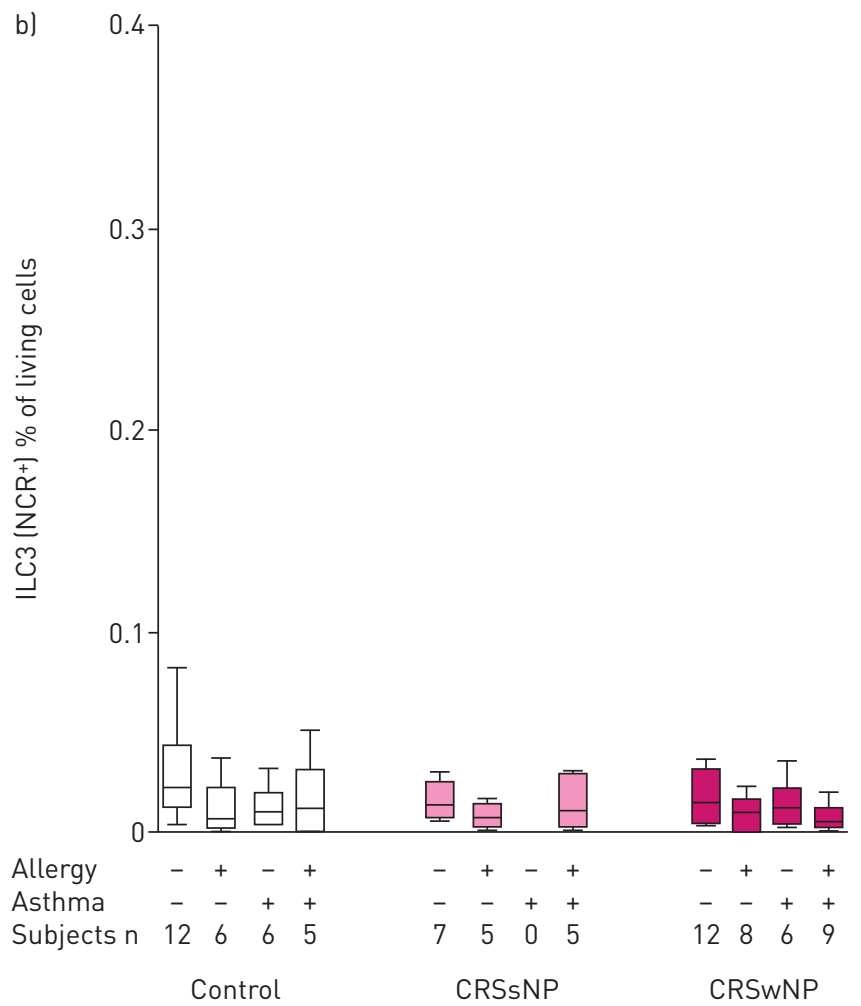

FIGURE 4 Relative number of a) natural cytotoxicity receptor-negative (NCR ${ }^{-}$) and b) -positive (NCR ) type 3 innate lymphoid cells (ILC3s) in controls, patients with chronic rhinosinusitis without nasal polyps (CRSsNP) and with nasal polyps (CRSwNP) separated on comorbidity status. Data are presented as percentage ILC3s of total living cells, and presented as box-and-whisker plots showing the minimum and the maximum value, the lower and the upper quartile, and the median. - : absent; +: present. *: $p<0.05$ versus controls.

ILC2 levels, differentiating the CRSwNP patient group on the basis of the different comorbidities indicates that the ILC2 population is not implicated in the pathophysiology of CRSwNP per se. Indeed, the levels of ILC2s in the mucosa of CRSwNP patients without comorbid allergy and/or asthma are not higher than in controls. However, ILC2 levels are increased in the inflamed mucosa of CRSwNP patients suffering from comorbid allergy and/or asthma. From these results, we speculate that previous studies, in which the authors oppositely claimed striking amounts of ILC2s in inflamed nasal polyps [9-11], included mainly CRSwNP patients suffering from comorbid allergy and/or asthma, but these clinical data were not communicated. Our results emphasise the importance of detailed reporting and analysis of the patients' clinical data in order to make correct interpretations.

In contrast to CRSwNP, CRSsNP is characterised by a predominant Th1 milieu with pronounced levels of IFN $-\gamma$ in the inflamed ethmoidal mucosa, histologically showing a fibrotic status of the inflamed tissue [1] with increased levels of transforming growth factor (TGF)- $\beta$ as a critical factor involved in this remodelling process [18]. Despite this Th1 signature, we here show that the mucosa of CRSsNP patients shows similar levels of ILC1s as observed for control subjects. Notably, the mucosa of CRSsNP patients retains clearly increased levels of ILC2s, but, in contrast to CRSwNP, the different comorbidities play no role therein. Recently, FoRKel et al. [19] reported a role for ILC2s in human liver fibrosis, which corroborates a previously suggested mechanism involving ILC2s in fibrosis development in the mouse liver [20]. These functional characteristics of ILC2s might be brought in agreement with the fibrotic status of the inflamed CRSsNP tissue, providing a possible functional explanation for the increased levels of ILC2s in CRSsNP. Indeed, it has been reported that TGF- $\beta$, an important mediator in the generation of fibrosis, enhances migration of ILC2s [21], supporting the increased presence of ILC2s in a Th1 disease such as CRSsNP. Moreover, TGF- $\beta$ has been reported to directly inhibit IL-33-induced IL-5 and IL-13 production in ILC2s [22, 23].

The question arises why ILC2s do not contribute to fibrosis in CRSwNP who display an oedematous status. We propose that ILC2s in CRSsNP do not possess tissue-residency phenotypic properties identical to those present in CRSwNP tissue. Indeed, while CD117- ILC2 can be found in controls and CRSsNP and CRSwNP patient groups, our data show that the CD117 ${ }^{+}$ILC2 population is virtually absent in controls and CRSsNP, whereas it is explicitly present in the inflamed mucosa of CRSwNP patients. Thus, the increased levels of the $\mathrm{CD} 117^{+}$ILC2s can be attributed to CRSwNP pathology, yielding the most pronounced expansion in 


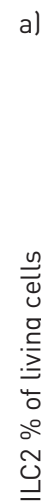
$\square \mathrm{CD} 117^{+}$

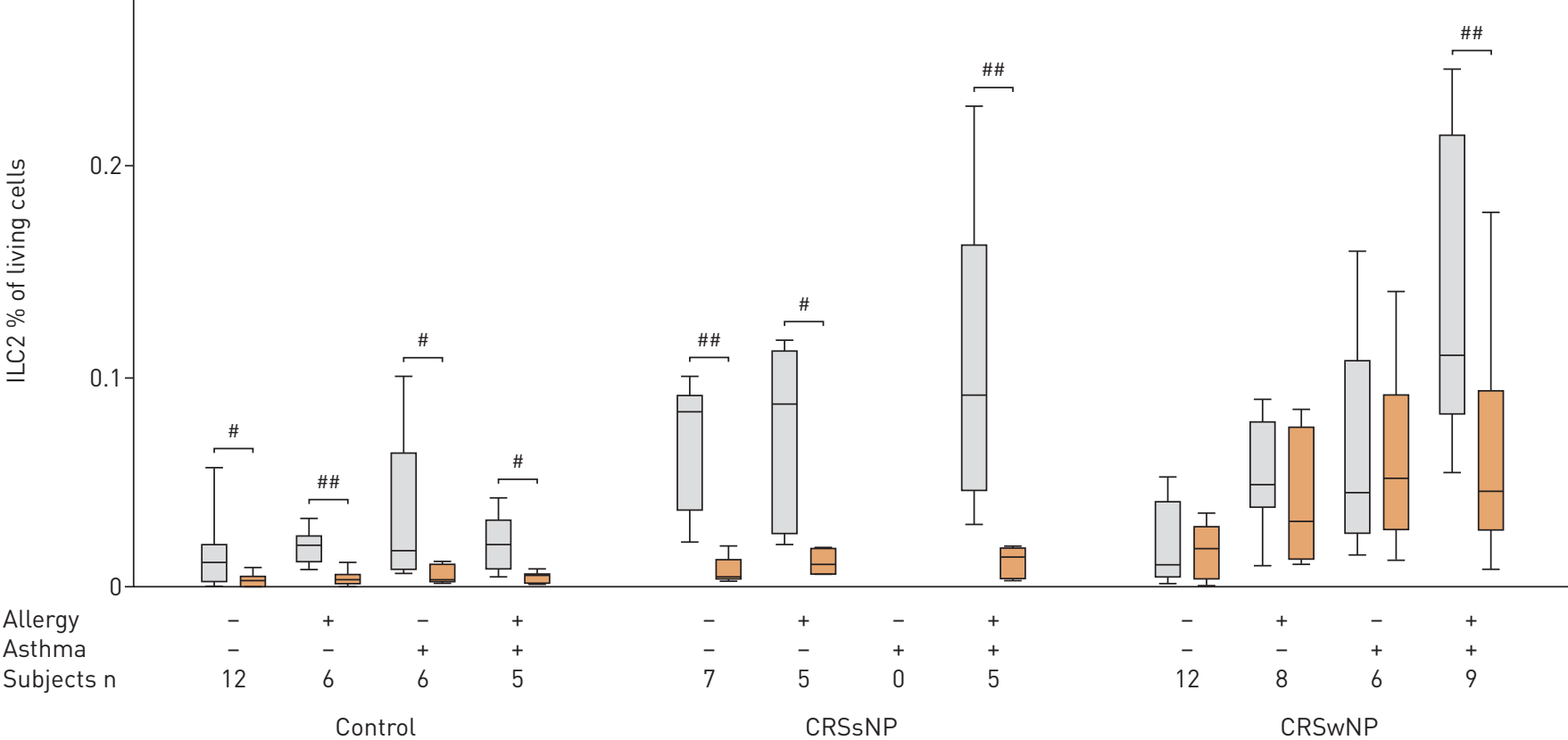

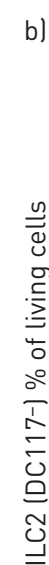

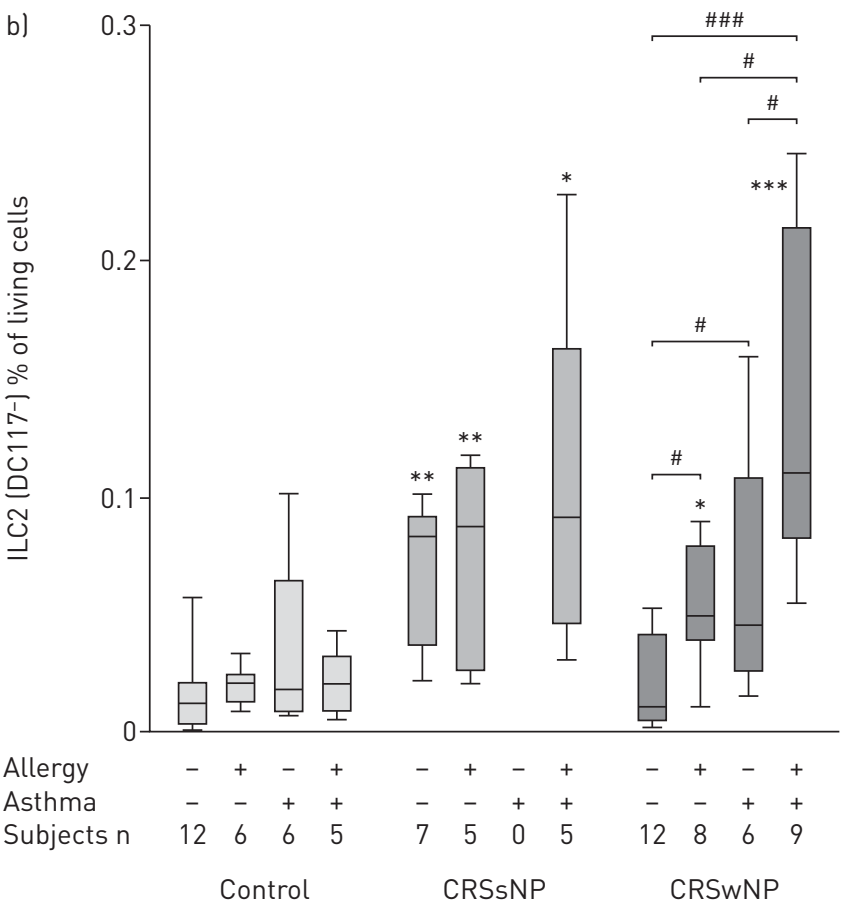

0.20

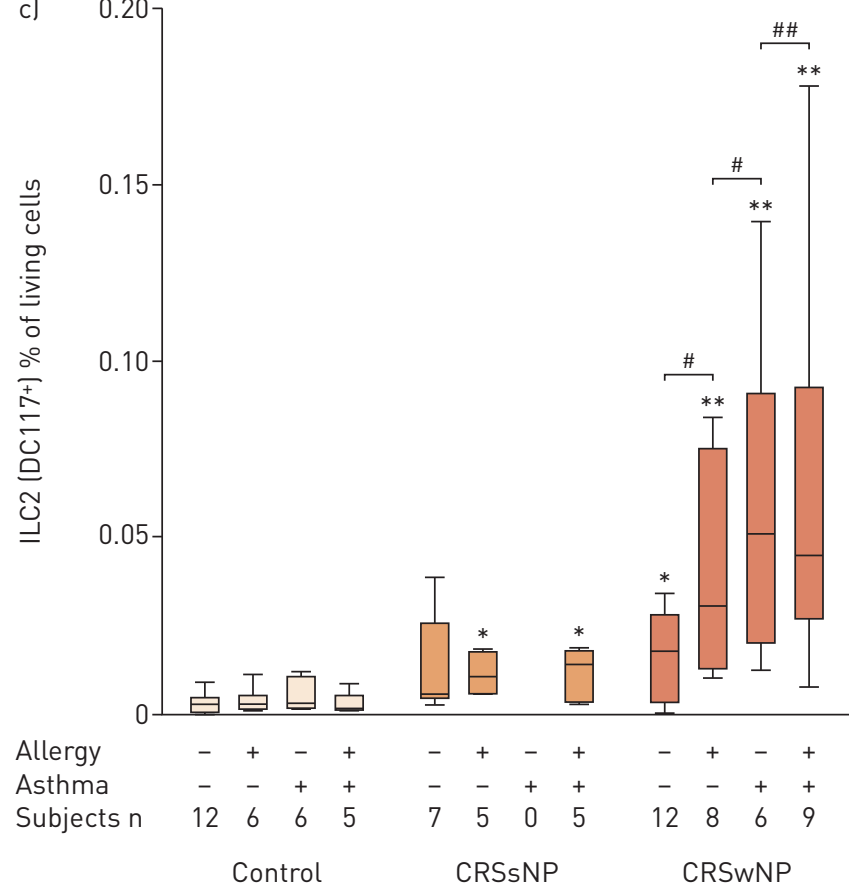

FIGURE 5 Relative number of a) and b) CD117- type 2 innate lymphoid cells (ILC2s) and a) and C) CD117 ILC2s in controls, patients with chronic rhinosinusitis without nasal polyps (CRSsNP) and with nasal polyps (CRSwNP) separated on comorbidity status. Values are expressed as percentage ILC2s of total living cells, and presented as box-and-whisker plots showing the minimum and the maximum value, the lower and the upper quartile, and the median. -: absent; +: present. ${ }^{*}: p<0.05 ;{ }^{* *}: p<0.01 ;{ }^{* * *}: p<0.001$ versus controls; ${ }^{\#}: p<0.05 ;{ }^{\# \#}: p<0.01 ;{ }^{\# \#}: p<0.001$ versus the indicated subject group.

combination with allergy or asthma. With regard to the functional implications for ILC2s in CRSwNP, CD117 (also known as c-kit) is a receptor for stem cell factor, best known for its essential role in haematopoietic stem cell proliferation, survival and adhesion [24]. In addition, in a setting of diminished c-kit signals in mice, ILC2s fail to develop [25]. In contrast to mice, in which mature ILC2s are reported to be exclusively CD117 [4], in humans both $\mathrm{CD}_{11} 7^{+}$and $\mathrm{CD}_{117^{-}}$ILC2 populations are observed [4, 5], making functional interpretations less straightforward. Lim et al. [26] observed no different levels in IL-13 production after PMA/ 


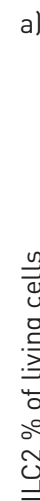

$0.3 \square \square \mathrm{IL} 1 \mathrm{RI} \mathrm{I}^{-}$
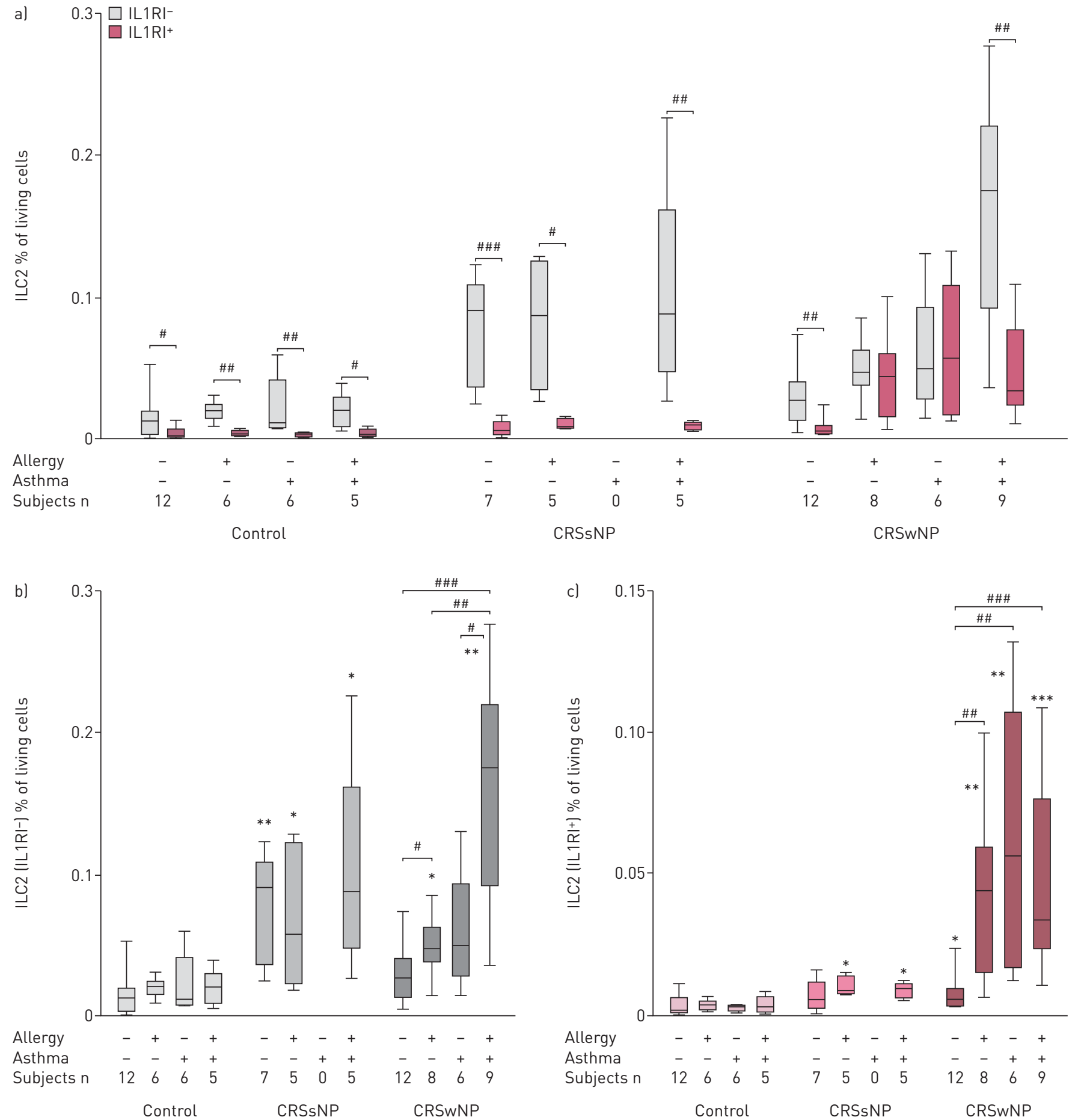

FIGURE 6 Relative number of a) and b) interleukin-receptor type I (IL-1RI) ${ }^{-}$type 2 innate lymphoid cells (ILC2s) and a) and c) IL-1RI ${ }^{+}$ILC2s in controls, patients with chronic rhinosinusitis without nasal polyps (CRSsNP) and with nasal polyps (CRSwNP) separated on comorbidity status. Data are presented as percentage ILC2s of total living cells, and presented as box-and-whisker plots showing the minimum and the maximum value, the lower and the upper quartile, and the median. - : absent; + : present. ${ }^{*}: p<0.05 ;{ }^{* *}: p<0.01 ;{ }^{* * *}$ : $p<0.001$ versus controls; ${ }^{\#}$ : $p<0.05$; $\#$ : $p<0.01 ;{ }^{\# \# \#}: p<0.001$ versus the indicated subject group.

ionomycin stimulation of cultured blood-derived CD117 $7^{-}$and $\mathrm{CD} 117^{+}$fractions of ILC2s, but the authors also reported that the expression of CD117 is affected by the culturing conditions, in which $\mathrm{CD} 117^{-}$cells can gain expression of CD117 and vice versa. Our results show that the phenotype of the native ex vivo ILC2s is already altered after $3 \mathrm{~h}$ culture without active stimulation; ILC2s derived from CRSsNP (natively lacking CD117 expression) develop a $\mathrm{CD} 117^{+}$fraction similar to that natively observed in CRSwNP, while CD117 ${ }^{+}$ILC2s derived from CRSwNP tissue downregulate surface CD117 and CRTH2 expression upon stimulation with 
a)
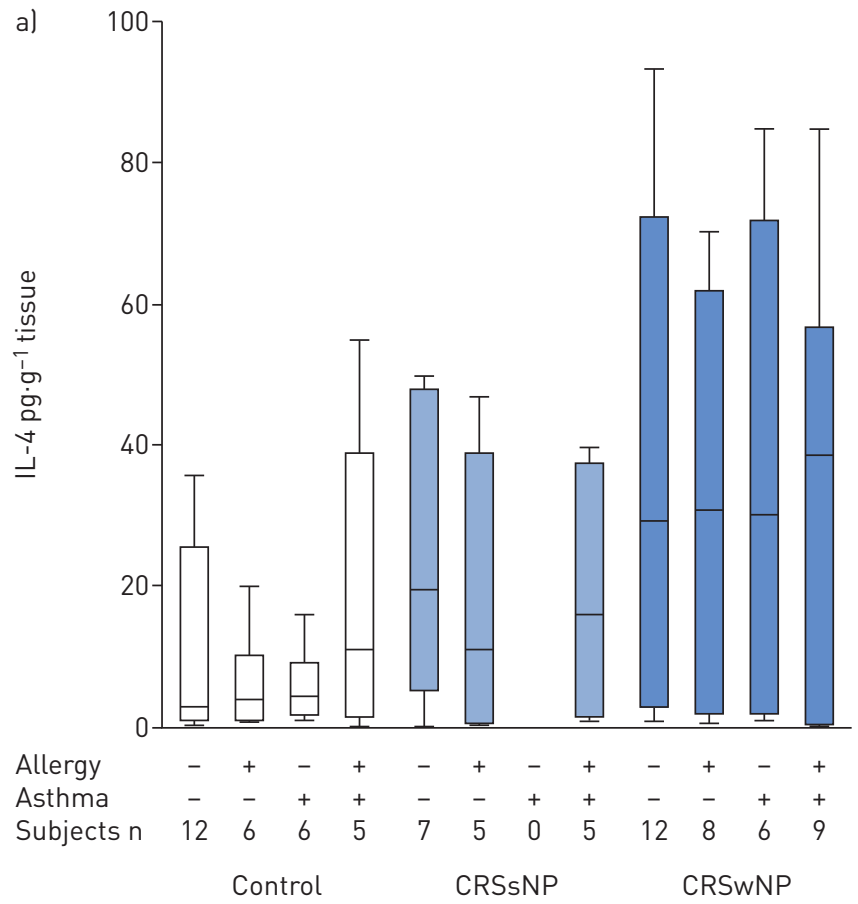

c)

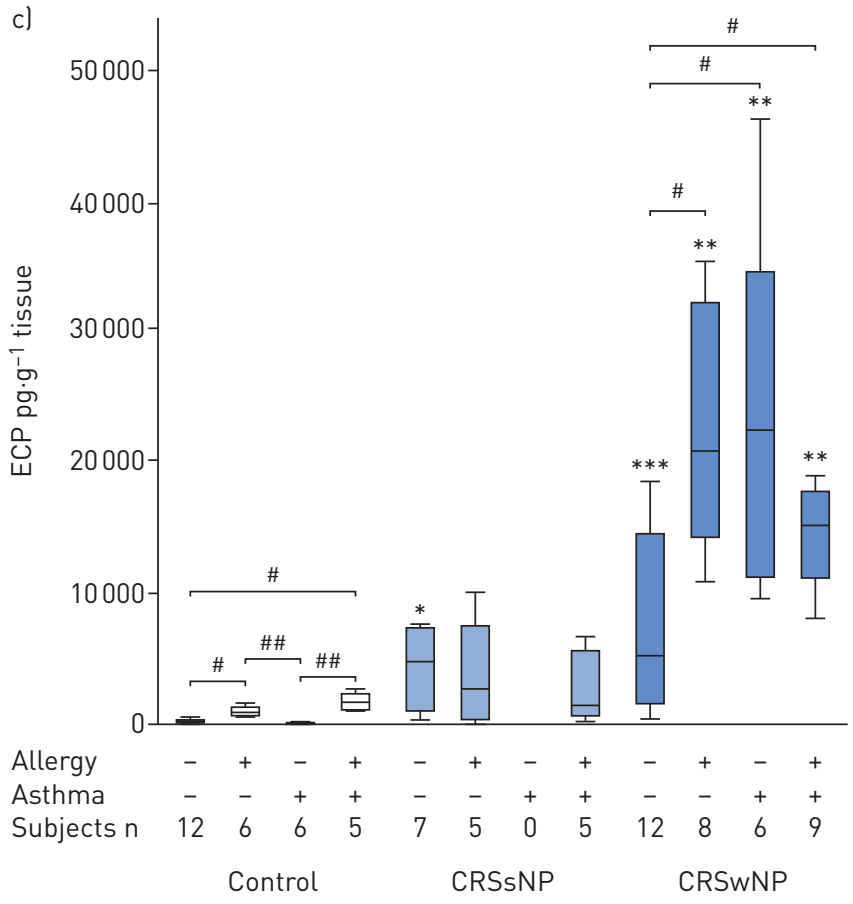

b)

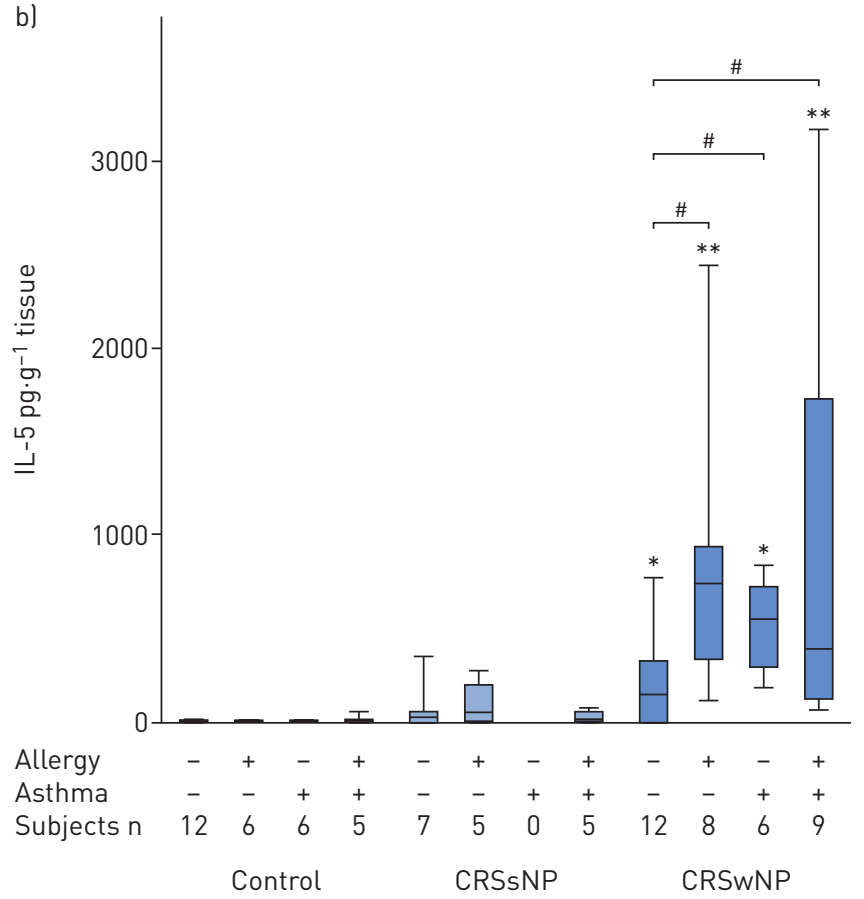

d)

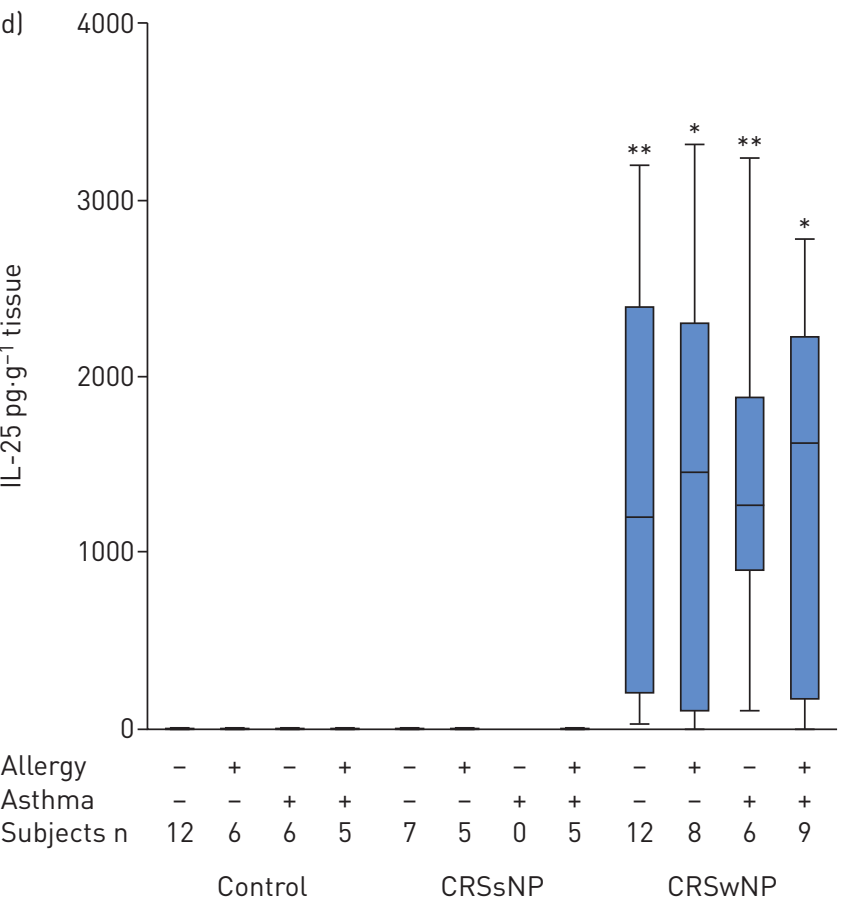

FIGURE 7 Quantification of tissue a) interleukin (IL)-4, b) IL-5, c) eosinophil cationic protein (ECP) and d) IL-25 protein levels by means of Luminex multiplex (a, b and d) or UniCAP (c) in controls, patients with chronic rhinosinusitis without nasal polyps (CRSsNP) and with nasal polyps (CRSwNP) separated on comorbidity status. Data are presented as box-and-whisker plots showing the minimum and the maximum value, the lower and the upper quartile, and the median. -: absent; +: present. ${ }^{*}: p<0.05 ;{ }^{* *}: p<0.01 ;{ }^{* * *}: p<0.001$ versus controls; ${ }^{\#}$ : $p<0.05 ;{ }^{\# \#}$ : $p<0.01$; versus the indicated subject group.

PMA/ionomycin. These results show that the surface expression status of ILC2s relies on the specific local tissue conditions inherent to the different pathologies and that the characteristics of the ILCs in different pathologies cannot be accurately assessed by means of in vitro approaches using cultured cells and artificial stimulation methods. Additionally, recent findings by Li et al. [27] on murine ILC2s indicate that ILC2s are phenotypically more heterogeneous than previously thought, whereby surface expression levels of various 
markers frequently used to identify ILC2s were dependent on their mode of activation, highly variable over time and differed between tissue compartments. Furthermore, when carefully inspecting the data from BAL et al. [10], the ILC2 population obtained upon culturing of blood-derived ILC2s was found to be entirely $\mathrm{CD} 117^{+}$. Moreover, these CD117 ${ }^{+}$ILC2s were previously regarded by the same research group to be less responsive in comparison to CD117 ${ }^{-}$ILC2s [9]. However, when the culture medium in their experiments was supplemented by IL-25, an equal amount of IL-13 was produced by both cell populations [9]. Because our data show that the levels of IL-25 are exclusively massively increased in the mucosa of CRSwNP patients, it can be assumed that the tissue-specific environment of CRSwNP likewise yields functionally active CD117 ${ }^{+}$ILC2s.

A second important phenotypic distinction between ILC2s present in CRSwNP and those in CRSsNP mucosa was found in the expression status of IL-1RI, the active receptor for IL1 $\beta$. While CRSsNP and control tissues lack ILC2s that express the active IL-1 $\beta$ receptor, IL-1RI ${ }^{+}$ILC2s are clearly present in CRSwNP, where they are particularly high in patients with comorbid allergy or asthma. ILC2s were initially thought to unambiguously express IL-1RI $[3,5]$, but in an article that assessed the heterogeneity of human $\mathrm{CD} 127^{+}$innate lymphoid cells by means of single-cell RNA sequencing, BJöRKLUND et al. [13] observed no expression of IL-1RI in ILC2s of uninflamed tonsil tissue. We report that in the mucosa of human upper airways, the level of IL-1RI expression in ILC2s is dependent on the specific local tissue conditions inherent to the different pathologies or the normal mucosal airway physiology. Indeed, in controls and CRSsNP, virtually all ILC2s lack the expression of IL-1RI, regardless of the absence or presence of any comorbidities. In the CRSwNP patient group, IL-1RI expression is clearly higher in patients with comorbid allergy and/or asthma. Because ILC2 cells derived from peripheral blood already have a substantial surface expression of IL-1R1 [15], our data (corroborated by the lack of IL-1RI expression in uninflamed tonsil tissue as reported by BJÖRKLUND et al. [13]) allows us to postulate that IL-1RI expression is downregulated in ILC2s at local tissue level under normal physiology and CRSsNP, probably by the lack of a specific trigger that maintains IL-1RI expression or by the presence of a trigger that downregulates its expression. Alternatively, the local mucosal tissue milieu of controls and CRsNP might only attract IL-1RI ${ }^{-}$ILC2s from the blood while the tissue inflammatory environment in CRSwNP enables the chemotaxis of the IL-1RI ${ }^{+}$subset. Regardless of which rationale might be valid, parabiosis experiments indeed showed that ILCs residing in the nonlymphoid tissues are primarily maintained through self-renewal with minimal contribution from haematogenous precursors and do not redistribute systemically [28], further corroborating that the local tissue environment is able to fine-tune the different ILC subsets into their final phenotype. What exactly triggers this clear-cut expression of the IL-1RI in CRSwNP, but not in CRSsNP, remains for further investigation. Functionally, IL-1RI engagement by IL-1 $\beta$ could directly affect human ILC2s by inducing low expression of the transcription factor T-BET and the cytokine receptor chain IL-12R $\beta 2$, which subsequently allows IL-12 to induce the conversion of these activated ILC2s into an ILC1 phenotype [10, 15]. As we could not detect measurable IL-12 levels in this tissue under any disease status, the importance of this functional plasticity in the human upper airway mucosa turns out to be unsure. Phenotypically, IL-1 $\beta$ is reported to significantly enhance expression of the receptors for IL-25, IL-33 and TSLP on ILC2 cells and to potentiate their responses to these cytokines. IL-1 $\beta$-treated ILC2 cells produce significantly more Th2

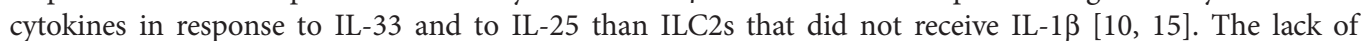
IL-1RI expression on ILC2s in CRSsNP and its presence on ILC2s in CRSwNP is in agreement with the absence and presence of substantial IL-5 production in the respective disease status.

When comparing the levels of ILC2s with the levels of Th2 mediators in our study, it is clear that there is no relationship between the numbers of CD117 ${ }^{-}$IL-1RI ${ }^{-}$ILC2s and the concentration shifts of IL-5 and ECP in the different disease states and comorbidities. Conversely, CD117 $7^{+} \mathrm{IL}-1 \mathrm{RI}^{+} \mathrm{ILC} 2 \mathrm{~s}$ are the most likely to have contributed to the shaping of the Th2 environment in CRSwNP, as could be concluded from the observation that this ILC2 subset clearly follows the pattern of IL-5 levels and the number of eosinophils that were assessed by means of ECP concentrations. The latter data corroborate a previous study that showed an interrelationship between eosinophilia and ILC2 levels in CRSwNP patients [29]. Our data show in addition that this is probably primarily due to CD117 ${ }^{+}$IL-1RI ${ }^{+}$ILC2s, which explains the lower eosinophil levels in CRSsNP in comparison to CRSwNP, despite the increased levels of total ILC2 population, being mainly CD117 ${ }^{-} \mathrm{IL}^{-1 R I^{-}}$in CRSsNP.

\section{Conclusion}

We have shown that the three ILC subsets are present in human upper airway mucosa. In noninflamed conditions, ILC1s are the most prominent subset. In CRSwNP, ILC1s are decreased while ILC2s are not increased. However, focusing on the $\mathrm{CD}_{117^{+}}$and $\mathrm{IL}-1 \mathrm{RI}^{+}$fractions within the ILC2s, and taking comorbidities into account, we unveil the precise contribution of the ILC2s in CRSwNP. We describe that only CD $117^{+}$IL-1RI $I^{+}$ILC2s (exclusively present in CRSwNP) possess an interrelationship with Th2 cytokine and eosinophil levels in human upper airway mucosa. Indeed, in CRSsNP, mainly CD117 ${ }^{-}$IL-1RI $^{-}$ILC2s are increased, yielding lower eosinophilia in this disease despite the high levels of ILC2s. Moreover, these data 
unveil for the first time that the $\mathrm{CD} 117^{-}$and the $\mathrm{CD} 117^{+}$fractions within the human ILC2 population are not just random phenomena and that IL-1RI expression is not ubiquitous in ILC2s. Both are affected by local tissue conditions present under the specific inflammatory conditions. The specific ILC2 expression status could in turn further shape the tissue-specific environment as a consequence of the possible functional implications on CD117 and IL-1RI expression on the inflammatory mediator release by ILC2s.

Conflict of interest: None declared.

Support statement: This work was supported by funding from a Concerted Research Action project (01G01317) from the Special Research Fund of Ghent University and from the Fund for Scientific Research (FWO) (G.0444.17N). S. Taveirne is supported by the FWO (12N4515N). Funding information for this article has been deposited with the Crossref Funder Registry.

\section{References}

1 Van Crombruggen K, Zhang N, Gevaert P, et al. Pathogenesis of chronic rhinosinusitis: inflammation. J Allergy Clin Immunol 2011; 128: 728-732.

2 Bachert C, Zhang N, Holtappels G, et al. Presence of IL-5 protein and IgE antibodies to staphylococcal enterotoxins in nasal polyps is associated with comorbid asthma. J Allergy Clin Immunol 2010; 126: 962-968. Sonnenberg GF, Mjösberg J, Spits H, et al. SnapShot: innate lymphoid cells. Immunity 2013; 39: 622.

4 Spits H, Artis D, Colonna M, et al. Innate lymphoid cells - a proposal for uniform nomenclature. Nat Rev Immunol 2013; 13: 145-149.

5 Mjösberg J, Spits H. Human innate lymphoid cells. J Allergy Clin Immunol 2016; 138: 1265-1276.

6 Bernink JH, Peters CP, Munneke M, et al. Human type 1 innate lymphoid cells accumulate in inflamed mucosal tissues. Nat Immunol 2013; 14: 221-229.

7 Morita H, Moro K, Koyasu S. Innate lymphoid cells in allergic and nonallergic inflammation. J Allergy Clin Immunol 2016; 138: 1253-1264.

8 Eberl G, Marmon S, Sunshine MJ, et al. An essential function for the nuclear receptor ROR $\gamma \mathrm{t}$ in the generation of fetal lymphoid tissue inducer cells. Nat Immunol 2004; 5: 64-73.

9 Mjösberg JM, Trifari S, Crellin NK, et al. Human IL-25- and IL-33-responsive type 2 innate lymphoid cells are defined by expression of CRTH2 and CD161. Nat Immunol 2011; 12: 1055-1062.

10 Bal SM, Bernink JH, Nagasawa M, et al. IL-1 $\beta$, IL-4 and IL-12 control the fate of group 2 innate lymphoid cells in human airway inflammation in the lungs. Nat Immunol 2016; 17: 636-645.

11 Mjösberg J, Bernink J, Golebski K, et al. The transcription factor GATA3 is essential for the function of human type 2 innate lymphoid cells. Immunity 2012; 37: 649-659.

12 Hazenberg MD, Spits H. Human innate lymphoid cells. Blood 2014; 124: 700-709.

13 Björklund AK, Forkel M, Picelli S, et al. The heterogeneity of human CD127 $7^{+}$innate lymphoid cells revealed by single-cell RNA sequencing. Nat Immunol 2016; 17: 451-460.

14 Silver JS, Kearley J, Copenhaver AM, et al. Inflammatory triggers associated with exacerbations of COPD orchestrate plasticity of group 2 innate lymphoid cells in the lungs. Nat Immunol 2016; 17: 626-635.

15 Ohne Y, Silver JS, Thompson-Snipes L, et al. IL-1 is a critical regulator of group 2 innate lymphoid cell function and plasticity. Nat Immunol 2016; 17: 646-655.

16 Buonocore S, Ahern PP, Uhlig HH, et al. Innate lymphoid cells drive interleukin-23-dependent innate intestinal pathology. Nature 2010; 464: 1371-1375.

17 Klose CS, Kiss EA, Schwierzeck V, et al. A T-bet gradient controls the fate and function of CCR6-ROR $\gamma \mathrm{t}^{+}$innate lymphoid cells. Nature 2013; 494: 261-265.

18 Van Bruaene N, Derycke L, Perez-Novo CA, et al. TGF- $\beta$ signaling and collagen deposition in chronic rhinosinusitis. J Allergy Clin Immunol 2009; 124: 253-259.

19 Forkel M, Berglin L, Kekäläinen E, et al. Composition and functionality of the intrahepatic innate lymphoid cell-compartment in human non-fibrotic and fibrotic livers. Eur J Immunol 2017; 47: 1280-1294.

20 McHedlidze T, Waldner M, Zopf S, et al. Interleukin-33-dependent innate lymphoid cells mediate hepatic fibrosis. Immunity 2013; 39: 357-371.

21 Denney L, Byrne AJ, Shea TJ, et al. Pulmonary epithelial cell-derived cytokine TGF- $\beta 1$ is a critical cofactor for enhanced innate lymphoid cell function. Immunity 2015; 43: 945-958.

22 Doherty TA, Broide DH. Pathways to limit group 2 innate lymphoid cell activation. J Allergy Clin Immunol 2017; 139: 1465-1467.

23 Rigas D, Lewis G, Aron JL, et al. Type 2 innate lymphoid cell suppression by regulatory $\mathrm{T}$ cells attenuates airway hyperreactivity and requires inducible T-cell costimulatory-inducible T-cell costimulator ligand interaction. J Allergy Clin Immunol 2017; 139: 1468-1477.

24 Roberts R, Govender D. Gene of the month: KIT. J Clin Pathol 2015; 68: 671-674.

25 Russi AE, Walker-Caulfield ME, Ebel ME, et al. Cutting edge: c-Kit signaling differentially regulates type 2 innate lymphoid cell accumulation and susceptibility to central nervous system demyelination in male and female SJL mice. J Immunol 2015; 194: 5609-5613.

26 Lim AI, Menegatti S, Bustamante J, et al. IL-12 drives functional plasticity of human group 2 innate lymphoid cells. J Exp Med 2016; 213: 569-583.

27 Li BWS, Stadhouders R, de Bruijn MJW, et al. Group 2 innate lymphoid cells exhibit a dynamic phenotype in allergic airway inflammation. Front Immunol 2017; 8: 1684

28 Gasteiger G, Fan X, Dikiy S, et al. Tissue residency of innate lymphoid cells in lymphoid and nonlymphoid organs. Science 2015; 350: 981-985.

29 Walford HH, Lund SJ, Baum RE, et al. Increased ILC2s in the eosinophilic nasal polyp endotype are associated with corticosteroid responsiveness. Clin Immunol 2014; 155: 126-135. 